\title{
El tratamiento con implantes dentales en los pacientes adultos mayores
}

\section{Dental implant treatment in elderly patients}

\author{
Velasco Ortega E*, Monsalve Guil L**, Jiménez Guerra A***, Segura Egea JJ****, \\ Matos Garrido N***, Moreno Muñoz J***
}

\section{RESUMEN}

En las últimas décadas, la implantología oral ha representado una técnica creciente en gerodontología. En este sentido, la edad avanzada no es una contraindicación para la implantología oral. Desde un punto de vista integral, es necesario la valoración de las condiciones médicas del paciente mayor, un diagnóstico oral, y una correcta planificación de tratamiento, con un estricto protocolo quirúrgico y prostodóncico. Muchos pacientes candidatos para la cirugía de implantes son mayores, y su perfil médico puede ser complejo. La comunicación con su médico puede ser importante para minimizar los riesgos quirúrgicos. Después de la fase quirúrgica, el éxito a largo plazo de los implantes dentales exige una buena reacción de los tejidos blandos y duros periimplantarios. Prótesis fijas y removibles pueden estar indicadas en los pacientes mayores edéntulos, según diferentes protocolos de carga funcional. El mantenimiento por el paciente y las revisiones periódicas por el dentista son muy importantes. Las prótesis implantosoportadas mejoran la calidad de vida oral y el bienestar psicosocial de los pacientes ancianos edéntulos.

Palabras clave: Implantes dentales, adultos mayores, oseointegración, pacientes geriátricos, implantología oral.

\section{SUMMARY}

During last decades, implant dentistry has been an increased technique in geriatric dentistry. Aging is not a contraindication for oral implantology. From a comprehensive point of view, are necessary a medical assessment of the aged patient, an oral diagnosis, a correct treatment planning, with a strict protocol of surgical and prosthetic procedures. Many patients for dental implant surgery are elderly, and their overall medical condition may be complex. Consultation with the patient's physician is important to ensure that surgical risks are minimized. After the surgical step, a good reaction of hard and soft tissues is necessary for long-term success of dental implants. Fixed and removable prostheses supported by dental implants were been indicated in edentulous older patients, according several functional loading protocols. Maintenance by the patient and periodical follow-up by the dentist are very important. Implant-supported prostheses improve oral quality of life and psychosocial well-being of edentulous aged patients.

Key words: Dental implants, elderly patients, osseointegration, geriatric patients, implant dentistry.

Fecha de recepción: 2 de mayo 2015.

Aceptado para publicación: 6 de mayo 2015.

* Profesor Titular de Odontología Integrada de Adultos y Gerodontología. Director del Máster de Implantología Oral. Facultad de Odontología. Universidad de Sevilla.

** Profesor Asociado de Odontología Integrada de Adultos y Gerodontología. Profesor del Máster de Implantología Oral. Facultad de Odontología. Universidad de Sevilla.

*** Profesor Colaborador Docente de Odontología Integrada de Adultos y de Gerodontología. Profesor del Máster de Implantología Oral. Facultad de Odontología. Universidad de Sevilla.

**** Catedrático de Patología y Terapéutica Dental. Profesor del Máster de Implantología Oral. Facultad de Odontología. Universidad de Sevilla.

Velasco Ortega E, Monsalve Guil L, Jiménez Guerra A, Segura Egea JJ, Matos Garrido N, Moreno Muñoz J. El tratamiento con implantes dentales en los pacientes adultos mayores. Av. Odontoestomatol 2015; 31 (3): 217-229. 


\section{INTRODUCCIÓN}

Clásicamente, la pérdida de dientes ha sido el estado dental más prevalente entre los pacientes mayores. Se ha aceptado, incluso, el edentulismo como un signo del envejecimiento humano por los profesionales de la medicina, la geriatría y la odontología. En este sentido, en los últimos 100 años el tratamiento dental más frecuente entre los adultos mayores ha consistido, fundamentalmente, en la extracción de los dientes naturales enfermos y su sustitución por los dientes artificiales protésicos (1-4).

En las últimas décadas, el uso de los implantes dentales ha alcanzado una importancia creciente en la práctica clínica de la odontología geriátrica o gerodontología. En este sentido, los implantes dentales pueden reemplazar la pérdida de uno, varios o todos los dientes en el paciente geriátrico. Sin embargo, la posibilidad de sustituir los dientes perdidos por estructuras protésicas sobre implantes ha representado una revolución en el tratamiento de los pacientes totalmente desdentados o edéntulos. El éxito a largo plazo de la rehabilitación oral de los maxilares edéntulos ha sido establecido mediante prótesis sobre implantes dentales (1-4).

La implantología oral ha experimentado un gran avance en los últimos años, ya que permite reemplazar los dientes perdidos con un alto grado de satisfacción a nivel funcional y estético. La pérdida parcial o total de los dientes no sólo afecta a la estética facial sino también a funciones tan vitales como son la masticación y la fonación . En este tipo de pacientes podemos realizar rehabilitaciones prostodóncicas con un alto porcentaje de éxito, que antes sólo se podía realizar con el tratamiento con una prótesis completa removible convencional, con el beneficio limitado que daba este tratamiento (1-4).

El gran desarrollo de la implantología oral, y su éxito constatado a largo plazo así como el beneficio que produce en los pacientes mayores, ha hecho posible que los profesionales de la salud oral podamos ofrecer esta alternativa de tratamiento en los pacientes desdentados totales. Es necesario comprender, que el éxito terapéutico con implantes dentales en el paciente edéntulo de edad avanzada depende de una valoración integral (salud general y oral) con una pre- via planificación del tratamiento, de un estricto protocolo quirúrgico y prostodóncico, y de la realización de un programa de seguimiento durante toda la vida (5).

\section{VALORACIÓN MÉDICA DEL PACIENTE GERIÁTRICO}

El tratamiento con implantes dentales en los pacientes mayores requiere un diagnóstico integral y una planificación de tratamiento para conseguir unos aceptables resultados funcionales y estéticos. Los factores médicos pueden jugar un importante papel en el tratamiento implantológico de los pacientes geriátricos porque los fenómenos biológicos de la oseointegración, a través del sistema óseo, están muy relacionados con la salud general del paciente. La mayor parte de las contraindicaciones generales clásicas absolutas en el tratamiento con implantes son cada vez más provisionales, ya que los diversos tratamientos médicos han mejorado la evolución clínica de las diversas condiciones sistémicas (ej.: diabetes, cardiopatías) mejorando la respuesta tisular y favoreciendo la terapéutica con implantes. Ahora bien, los estudios demuestran que en estos pacientes, generalmente, las tasas de éxito son menores. En efecto, en primer lugar la inserción de los implantes se realiza en un huésped con una respuesta tisular modificada por su enfermedad general; y en segundo lugar porque debido a la relación dinámica entre el estado sistémico del paciente y el mantenimiento de los implantes, es difícil conocer o prevenir la evolución de la enfermedad del paciente y todas las complicaciones futuras que pueden aparecer y que puedan afectar a la oseointegración (6).

En este sentido, el tratamiento con implantes oseointegrados constituye una terapéutica con unas expectativas en el paciente a largo plazo, que no consiste en la inserción de los implantes y su posterior rehabilitación prostodóncica en unos meses. Los resultados clínicos deben ser mantenidos en el tiempo y evaluados periódicamente, por lo que las relaciones entre la oseointegración y el estado sistémico del paciente son permanentes y pueden modificarse con la evolución de la enfermedad sistémica $(1,3,6)$.

Sin embargo, la primera consideración que debe establecerse en la valoración integral del paciente 
geriátrico con respecto al tratamiento implantológico, es que la edad avanzada no constituye per se ninguna contraindicación para que los pacientes mayores sean tratados con implantes dentales. La edad cronológica del paciente geriátrico no indica el estado funcional del paciente que debe ser establecido según su estado físico, mental y social, en comunicación directa con sus familia, médico de cabecera o geriatra para valorar los beneficios del tratamiento con implantes, sobre todo, en aquellos casos con posibles repercusiones médicas o legales $(1,3,6)$.

La edad avanzada no constituye un criterio de inclusión o exclusión del protocolo con implantes dentales. Cualquier paciente mayor, cuya salud general no excluya los procedimientos de cirugía oral menor o de cualquier protocolo prostodóncico, puede ser considerado como candidato al tratamiento con implantes. En estos casos, la limitación es definida por el grado de salud general y, sobre todo, por la capacidad funcional del paciente, sobre todo para realizar un correcto mantenimiento del tratamiento realizado (ej.: higiene oral) $(1,3,6)$.

Dicho de otro modo, la selección del paciente mayor es similar a la del paciente más joven. En este sentido, la evaluación del grado de salud general debe establecerse, de forma detallada, y puede contraindicar el tratamiento con implantes, cuando existe un compromiso médico grave o incapacitante. Los pacientes geriátricos con un estado físico o psíquico deteriorado no son buenos candidatos para un tratamiento con implantes dentales. Frecuentemente, el implantólogo debe consultar con el médico de cabecera o geriatra, para realizar una completa historia clínica, sobre todo, cuando el paciente padece alguna condición sistémica crónica, con consumo de múltiples medicamentos que pueda influir sobre la salud oral o represente una tendencia invalidante (6).

Los pacientes mayores tratados con implantes dentales que presentan enfermedades cardiovasculares no suelen presentar más complicaciones, siempre que esté controlado su estado general y las tasas de éxito suelen ser muy elevadas. No hay que olvidar que muchos medicamentos que se utilizan para la hipertensión y las enfermedades cardiovasculares pueden presentar efectos adversos orales (ej.: xeros- tomía, disgeusia, reacción liquenoide) que puede hacer necesario la modificación del plan de tratamiento, sobre todo quirúrgico (ej.: anticoagulantes, antiagregantes) (Figura 1) (7).

El tratamiento con implantes puede ser mantenido con éxito por los pacientes mayores a lo largo de su vida (8). En este sentido, un estudio canadiense compara los resultados clínicos de 39 pacientes mayores de 60 años (edad media de 66 años) con 190 implantes para soportar 45 prótesis y de 43 adultos jóvenes (edad media de 41 años) con 184 implantes para soportar 45 prótesis (8). Los pacientes fueron monitorizados durante un periodo de 4 a 16 años después de la carga funcional. Las indicaciones prostodóncicas más frecuentes fueron la rehabilitación fija (51\%) y las sobredentaduras (27\%). El éxito acumulativo mostró una diferencia no significativa del $92 \%$ en el grupo mayor y del $86,5 \%$ en el grupo más joven. La mayor parte de los fracasos ocurrieron antes o durante el primer año después de la carga funcional protésica. Aunque los pacientes mayores padecían o desarrollaron trastornos médicos, la oseointegración no se alteró por su estado sistémico, lo que sugiere que el éxito del tratamiento implantológico puede no afectarse por las enfermedades comunes asociadas al envejecimiento. Este estudio canadiense no sólo confirma la hipótesis de que no hay diferencias entre los adultos mayores y jóvenes para el éxito de la oseointegración; sino que además, los resultados sugerían una tendencia de mayor éxito entre los pacientes ancianos (8).

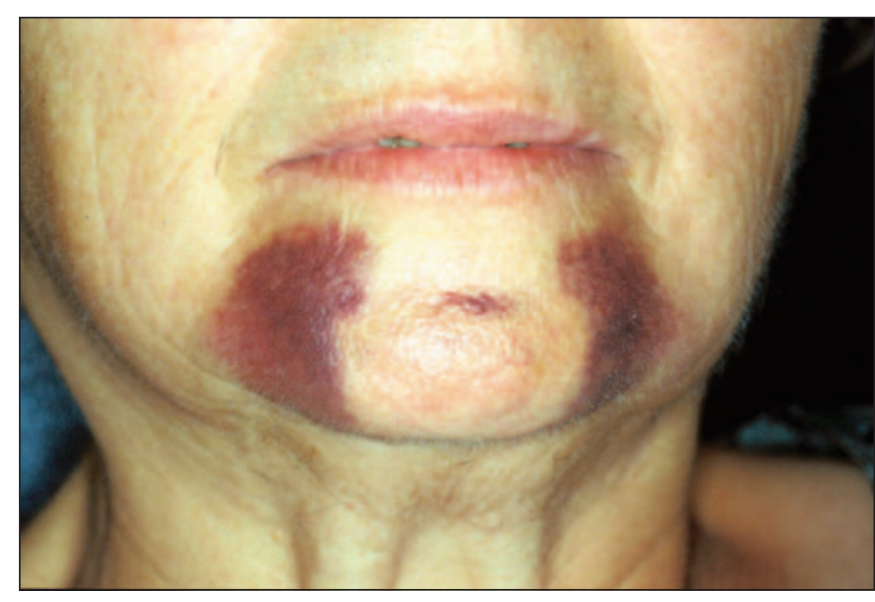

Fig. 1. Hematoma en localización de implantes para sobredentadura mandibular. 
La mayoría de los adultos mayores tratados con implantes son pacientes geriátricos que padecen enfermedades crónicas que suelen estar bien controladas $(9,10)$. Un estudio retrospectivo belga valora los resultados del tratamiento con implantes en pacientes mayores con una edad media de 60,8 \pm 10 ,2 años a lo largo de un periodo de 15 años (9). 495 pacientes fueron rehabilitados con sobredentaduras mandibulares sobre 1051 implantes. El 7,4\% de los pacientes presentaban alguna enfermedad crónica (diabetes, quimioterapia, radioterapia, enfermedad de Crohn) siendo la más frecuente la diabetes. El $12 \%$ de los pacientes eran fumadores. La tasa de éxito fue del 95,5\% después de 23 años. Los fracasos fueron más frecuentes significativamente entre los pacientes fumadores (27\% vs $12 \%$ ) (9).

Un estudio prospectivo canadiense valora los resultados del tratamiento con implantes en pacientes mayores con una edad media de 70 años a lo largo de un periodo de 15 años (10). 45 pacientes fueron rehabilitados con 47 sobredentaduras (42 mandibulares) sobre 132 implantes (115 mandibulares). El $69 \%$ de los pacientes presentaban alguna enfermedad crónica (cardíacas, endocrinas, artrosis, osteoporosis) siendo las más frecuentes las dolencias cardíacas. La tasa de éxito fue del 93,1\%. Los fracasos implantarios no estuvieron relacionados con los antecedentes médicos (10).

Los pacientes geriátricos diabéticos presentan frecuentemente más pérdida dental y periodontitis, y la diabetes es considerada como una condición de riesgo para los implantes dentales, ya que existe una menor capacidad de cicatrización debida a la enfermedad microvascular y a una deteriorada respuesta a la infección (11). En este sentido, la diabetes puede representar una contraindicación relativa para la cirugía de implantes. Sin embargo, los pacientes mayores que presentan un buen nivel de glucemia y están controlados pueden beneficiarse de la rehabilitación oral con implantes dentales y no suelen presentar una mayor tasa de fracasos implantarios (11).

Los grupos de pacientes tratados con implantes suelen incluir mujeres postmenopáusicas que padecen o han desarrollado osteoporosis. Aunque la osteoporosis provoca un descenso de la masa ósea corporal, sin embargo no contraindica el uso de los implantes, ya que no existe una correlación directa entre la densidad ósea a nivel maxilar o mandibular y el resto del esqueleto, probablemente debido al efecto beneficioso de la masticación (12). Un aspecto importante de la osteoporosis con respecto al tratamiento con implantes dentales está relacionado con el consumo de bisfosfonatos (13). En este sentido, la posibilidad de desarrollar osteonecrosis maxilar después de la cirugía de implantes está más relacionada con los bisfosfonatos administrados por vía intravenosa porque dificulta la capacidad reparadora ósea ante un traumatismo. En estos casos debe considerarse como una contraindicación absoluta (13).

Muchos pacientes mayores padecen cáncer y han sido tratados con radioterapia. Los efectos de la radioterapia sobre los implantes dentales ha sido estudiado en modelos animales y en serie de pacientes (14). Los estudios clínicos sugieren que los fracasos implantarios son entre 3 y 12 veces más frecuentes entre los pacientes irradiados. Estos fracasos están relacionados con dosis mayores de $50 \mathrm{~Gy}$, siendo más prevalente sobre todo en los implantes insertados en la maxilar superior (14). Aunque la inserción de implantes en pacientes geriátricos irradiados no está a largo plazo contraindicada, hay que tener en cuenta que predispone a una mayor pérdida de implantes. Es evidente que la cirugía de implantes debe ser realizada entre 6-12 meses después de la radioterapia, siendo más ideal entre 13-24 meses. Además, si se va a realizar una segunda cirugía de implantes para la colocación de los tornillos de cicatrización en los casos de técnica sumergida, debe esperarse no menos de 4-6 meses después de la inserción de los implantes (15).

Recientemente, se ha incorporado la posibilidad de realizar el tratamiento con implantes dentales en aquellos pacientes geriátricos que presentan enfermedades neurodegenerativas $(16,17)$. La sintomatología de la enfermedad de Parkinson se caracteriza por temblores, lentitud de movimientos y rigidez muscular. Se ha demostrado que el tratamiento con una prótesis sobre implantes mejora en estos pacientes su capacidad de llevar prótesis convencionales lo que provoca un impacto positivo sobre su bienestar integral (16). En estos pacientes es esencial la prevención de complicaciones y el mantenimiento de los tejidos periimplantarios, seleccionando el im- 
plantólogo protocolos fáciles de realizar con la inserción de un número reducido de implantes y prótesis removibles del tipo de las sobredentaduras (17). En los casos de pacientes con demencia o un deterioro significativo de su función cognitiva son muy importante los aspectos legales del tratamiento que debe incluir una información precisa a sus familiares directos (ej.: hijos), asistente social y a su médico o geriatra (17).

Otro factor de riesgo importante para el tratamiento implantológico en los adultos mayores lo constituye el consumo de tabaco (18). Existe una evidencia de una significativa asociación entre el hábito de fumar y los fracasos implantarios. Esta relación también se manifiesta en las localizaciones intervenidas con aumento de tejido óseo. Además el hábito de fumar incrementa la pérdida de hueso marginal y el desarrollo de periimplantitis (18). El consumo de tabaco en los adultos mayores está relacionado con una mayor incidencia de periodontitis (expresada por el nivel de pérdida ósea marginal ) y de enfermedad coronaria (19). Además del tabaco, los antecedentes de periodontitis son importantes en los pacientes mayores que han sido tratados con implantes y que pueden presentar tasas mayores de periimplantitis que los pacientes adultos más jóvenes porque inciden también otros factores como una menor visión o incapacidad manual para realizar un control adecuado de la placa bacteriana (20).

Una posible controversia del tratamiento con implantes dentales en los adultos mayores se deriva de su posible indicación clínica en aquellos pacientes geriátricos con un grado importante de dependencia o institucionalización (21-23). Un estudio sueco valora el tratamiento implantológico en pacientes mayores residentes en instituciones asistidas. Fueron examinados 3.310 pacientes de los que 35 tenían restauraciones sobre implantes (228 implantes con 40 rehabilitaciones fijas y 11 sobredentaduras). 12 de estos pacientes tenían una higiene oral buena o aceptable y 23 presentaban una higiene oral pobre o mala. No se observaron movilidad o fracturas de los implantes o de las prótesis. 15 implantes presentaban exposición vestibular de su superficie. La mayoría de los tratamientos tenían más de 15 años (21). En estos pacientes institucionalizados, es necesario un mantenimiento adecuado del tratamiento con implantes mediante programas específicos que hagan posible la revisión periódica de los implantes y de las prótesis correspondientes para evitar la incidencia de complicaciones biológicas y prostodóncicas (23).

La influencia de la salud oral sobre la salud general en los adultos mayores ha sido demostrada en algunos estudios que indican una mayor mortalidad en aquellos pacientes que han perdido un mayor número de dientes (24). En este sentido, recientemente, se ha publicado un estudio a 10 años, en que los pacientes mayores tratados con implantes presentan una mayor tasa de supervivencia que aquellos que no presentan tratamiento con implantes dentales, posiblemente debido al hecho de que los ancianos rehabilitados con implantes presentaban previamente un mejor grado de salud general que la población mayor normal. Es decir, la cohorte de adultos mayores que fueron tratados con implantes presentaban una mayor motivación, como consecuencia de su mejor estado sistémico, para rehabilitar sus dientes perdidos que los no tratados con implantes (25).

Un segundo estudio valora a pacientes edéntulos mandibulares tratados con implantes con diferentes protocolos quirúrgicos (técnica sumergida y no sumergida) e implantológicos (superficie mecanizada y superficie arenada). Entre los pacientes tratados, las enfermedades más frecuentes eran las cardiovasculares (28-56\%), la diabetes (3-13\%) y cáncer (1-12\%). El tabaco estaba presente en el 34-40\% de los pacientes. El estudio demuestra que los pacientes adultos tratados más jóvenes (edad media de 59,5 años en el momento de la cirugía) presentaron un significativo incremento de la mortalidad del $4,9 \%$ a los 5 años comparados con la población general; mientras que los pacientes adultos mayores (edad media de 76,6 años en el momento de la cirugía) presentaron un descenso significativo de la mortalidad del $6,8 \%(26)$.

\section{CIRUGÍA DE IMPLANTES}

Para realizar la cirugía de implantes en los pacientes mayores, sobre todo edéntulos totales, es necesario valorar los cambios ocasionados como consecuencia del edentulismo. Además de la exploración clíni- 
ca, los medios diagnósticos por imagen son imprescindibles, como la ortopantomografía o más recientemente, la tomografía computarizada de haz cónico (Figuras 2 y 3). Cuando el volumen óseo es adecuado o suficiente, la cirugía de inserción de implantes en los pacientes mayores no suele ofrecer complicaciones o desafíos importantes (6).

En este sentido, los pacientes mayores, previamente a la inserción de los implantes, deben ser medicados con prevención antibiótica el mismo día de la cirugía (ej.: amoxicilina + ácido clavulánico). Si el paciente presenta ansiedad por la intervención se le puede

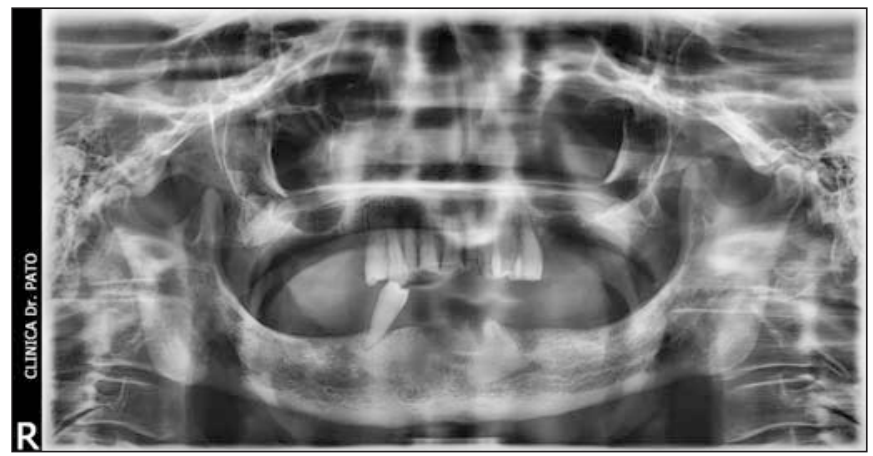

Fig. 2. Planificación de tratamiento con implantes mediante ortopantomografía. administrar un ansiolítico de acción corta (ej.: alprazolam). Es evidente que las normas generales de cirugía oral deben ser estrictamente establecidas (ej.: asepsia, anestesia, incisión, sutura) (6).

La técnica quirúrgica implantológica básica consiste en el fresado del hueso y la inserción del implante con una buena estabilidad primaria que consiga un buen contacto hueso-implante. El diseño macroscópico y la superficie tratada favorecen esta unión. El fresado del hueso que debe ser realizado cuidadosamente, con las fresas correspondientes estandarizadas, con irrigación con suero fisiológico, sin presión, y a velocidad reducida (alrededor de 800 r.p.m.), evita el sobrecalentamiento del lecho implantario que puede interferir la futura interfase tejido-implante. Tras la fase quirúrgica, durante dos o tres semanas, debe tenerse mucho cuidado con la utilización de la prótesis dental, especialmente entre los adultos mayores edéntulos totales. Posteriormente, se debe realizar la adaptación necesaria de la prótesis para evitar traumatismos sobre los implantes o tejidos vecinos (6).

En los casos más avanzados, el maxilar superior edéntulo sufre un proceso de reabsorción importante, sobre todo, en el sector posterior. Es frecuente

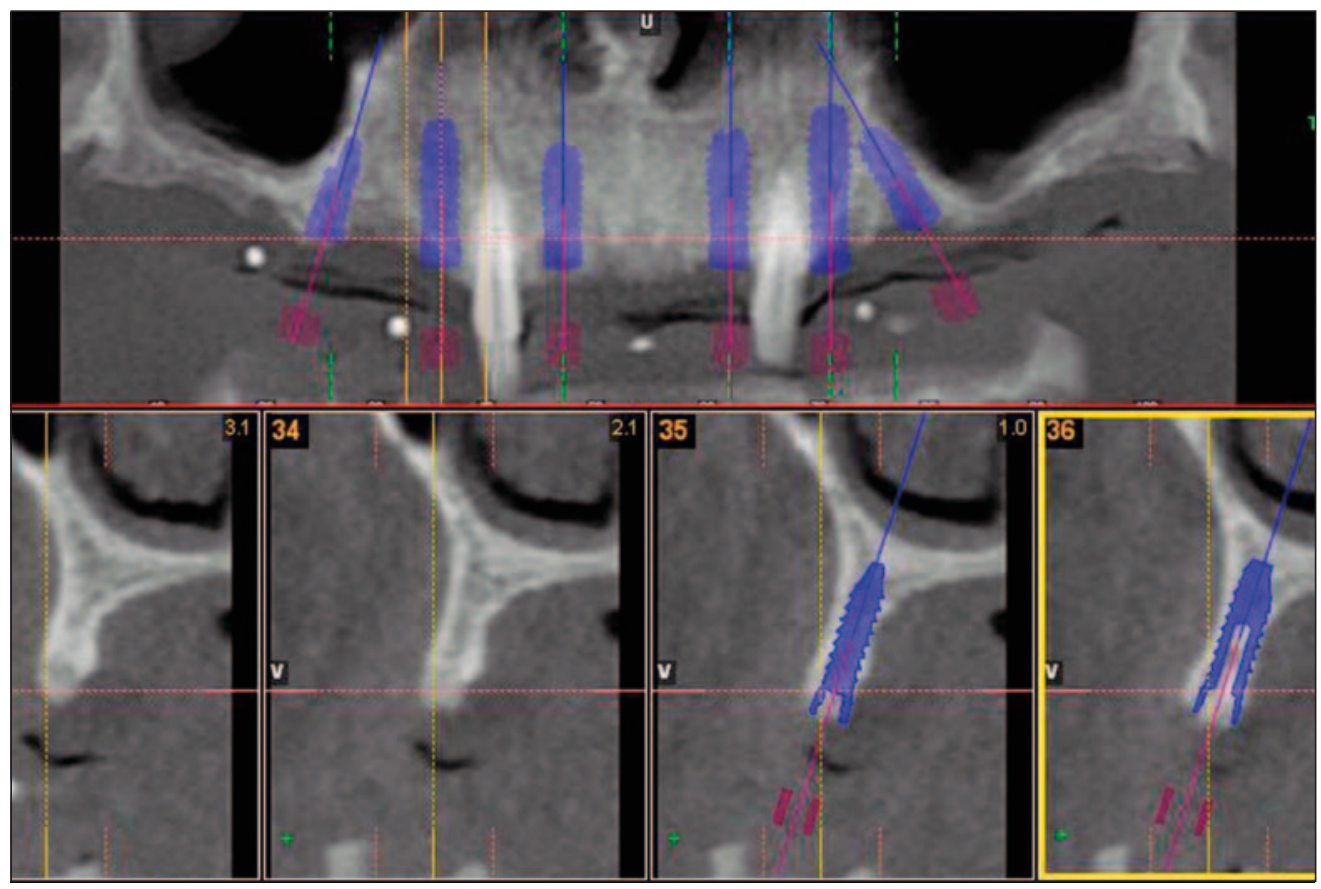

Fig. 3. Planificación quirúrgica de implantes en paciente edéntulo parcial mediante CBCT. 
que el seno maxilar obligue a la inserción de los implantes en el sector anterior, salvo que se recurra a la técnica de elevación del seno maxilar (Figura 4). En el maxilar atrófico con crestas óseas estrechas o hueso poco compacto se pueden utilizar una técnica poco traumática de ensanchamiento progresivo o expansión crestal, mediante la introducción de varios osteótomos de diámetro creciente, configurando un lecho óseo para la inserción del implante (27).

Los pacientes mayores con un proceso intenso de reabsorción maxilar necesitan una elevación de seno maxilar, generalmente bilateral, para la inserción de los implantes y su posterior rehabilitación prostodóncica, siendo a veces necesaria, la realización de injertos autólogos de cresta ilíaca, que mediante diferentes técnicas quirúrgicas pueden lograr un aceptable incremento del volumen óseo maxilar. En determinadas ocasiones, pueden ser recomendables los implantes cigomáticos (28).

La mandíbula atrófica presenta también retos importantes para el cirujano en relación al trayecto del dentario inferior. Los injertos autólogos han sido también muy utilizados, especialmente en la mandíbula, para obtener mayores volúmenes óseos sobre todo en sentido vertical y configurar un mejor pronóstico para los implantes. Estas técnicas de aumento del volumen óseo para la inserción de implantes requieren, frecuentemente, la utilización de materiales de injertos como pueden ser el propio hueso del paciente o substitutos óseos (29).

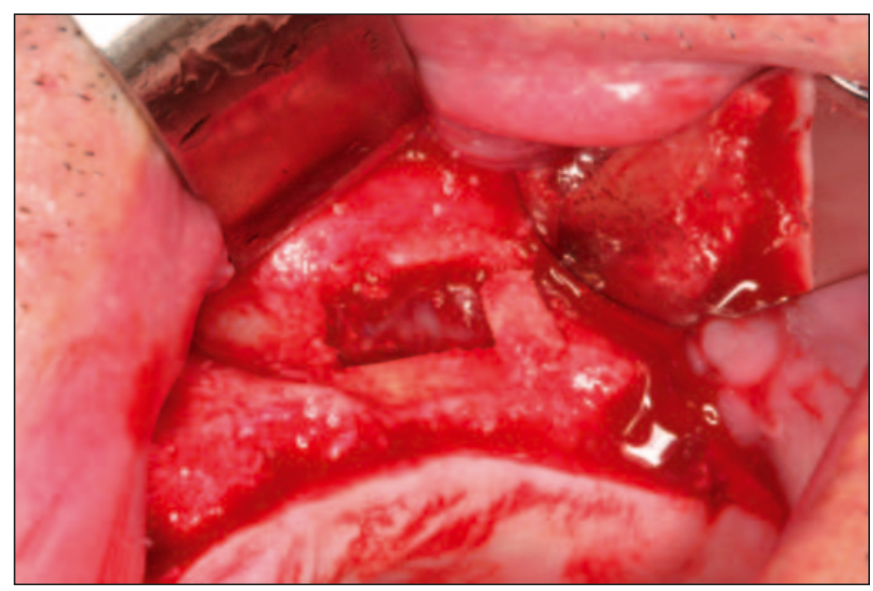

Fig. 4. Elevación del seno maxilar para la inserción de implantes.
Es evidente, que este protocolo de tratamiento implantológico necesita en los casos más complejos, anestesia general y hospitalización. El grado de morbilidad es importante ya que la cirugía de injertos ocasiona molestias tanto en la zona donante como en la receptora. Por lo tanto esta opción implantológica tiene que ser estrictamente valorada por el implantólogo teniendo en cuenta el perfil médico y farmacológico de los pacientes geriátricos para no incrementar el riesgo quirúrgico $(28,29)$.

Desde un punto de vista quirúrgico, el tratamiento con implantes en el adulto de edad avanzada está relacionado directamente con el diagnóstico individual del paciente y con el grado de edentulismo que presenta. Si el paciente presenta una pérdida dental parcial, las indicaciones serán implantes unitarios para coronas o varios implantes para la rehabilitación con puentes fijos. Sin embargo, si el paciente mayor es edéntulo total, el reto implantológico es más complejo, existiendo dos posibles alternativas rehabilitadoras sobre implantes, la sobredentadura y la rehabilitación fija (6).

La experiencia clínica en la rehabilitación de pacientes mayores con sobredentaduras demuestra que es recomendable la inserción de 2-4 implantes con una longitud mínima de 10 mm en los maxilares edéntulos. Es recomendable una distribución adecuada de los implantes que asegure mejor las cargas oclusales, especialmente en el maxilar superior (en número no inferior a 4 implantes), por lo que se debe insertar los implantes en la región anterior, lateral o premolar y posterior más que en el maxilar anterior. En contraste con el maxilar superior, la cirugía de implantes en las sobredentaduras mandibulares demuestra que la inserción de 2, 3 o 4 implantes en el sector anterior interforaminal puede ser suficiente para conseguir un tratamiento de éxito, ya que el número de fracasos de implantes es menor en la mandíbula $(1,3,6)$. En determinadas situaciones clínicas de reabsorción ósea severa con crestas estrechas, en pacientes edéntulos totales, los miniimplantes con un diámetro de 1,8-2,4 mm han sido utilizados con éxito para soportar sobredentaduras con un éxito elevado (30).

En todos los casos de rehabilitación fija con implantes en el paciente edéntulo total es imprescindible 
realizar una férula diagnóstica y quirúrgica para la correcta inserción de los implantes. Actualmente, con el desarrollo de los nuevos métodos computarizados por imagen, y de los protocolos quirúrgicos guiados, se puede sistematizar muy favorablemente todo el tratamiento implantológico con un alto grado de precisión y éxito (31).

Para rehabilitar a un paciente mayor edéntulo total con una prótesis completa fija es necesario realizar una correcta elección del número de implantes y de su distribución en la arcada edéntula (Figuras 5-8). La distribución de los implantes es muy importante para conseguir una buena distribución de las fuerzas oclusales tanto en el sector posterior como en sec-

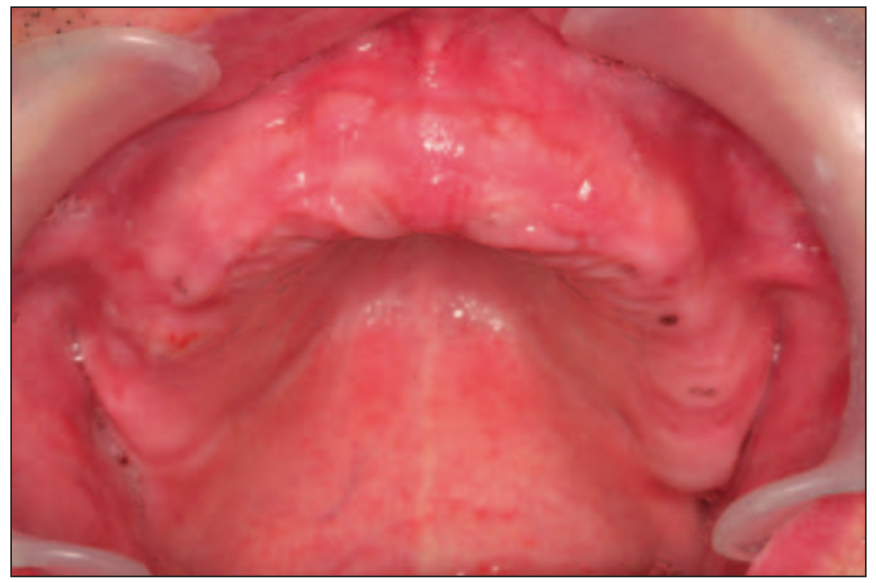

Fig. 5. Paciente geriátrico edéntulo total maxilar.

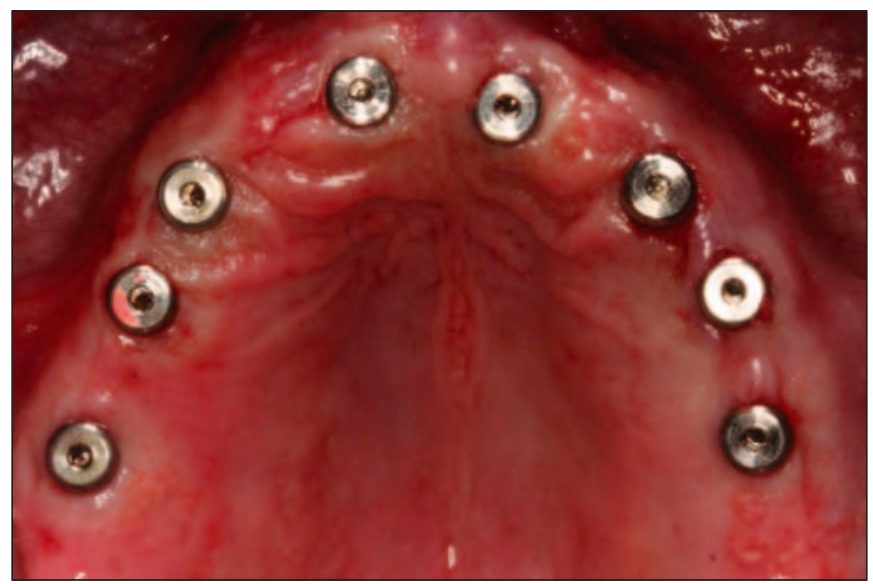

Fig. 6. Implantes insertados en el paciente geriátrico edéntulo total maxilar. tor anterior (Figuras 6 y 7). En general, se recomienda un número de 6-10 implantes teniendo en cuenta las disponibilidad ósea de los maxilares edéntulos, así como consideraciones oclusales y prostodóncicas (tipo de prótesis, materiales) especialmente en el maxilar superior $(32,32)$.

La longitud de los implantes puede constituir un factor importante en el éxito del tratamiento ya que se ha demostrado una relación directa entre una mayor longitud y unas mejores expectativas de éxito a largo plazo en los pacientes edéntulos completos. La longitud del implante permite una inserción estable y, en este sentido, asegurar al máximo la oseointegración ya que la superficie de contacto hueso/implante

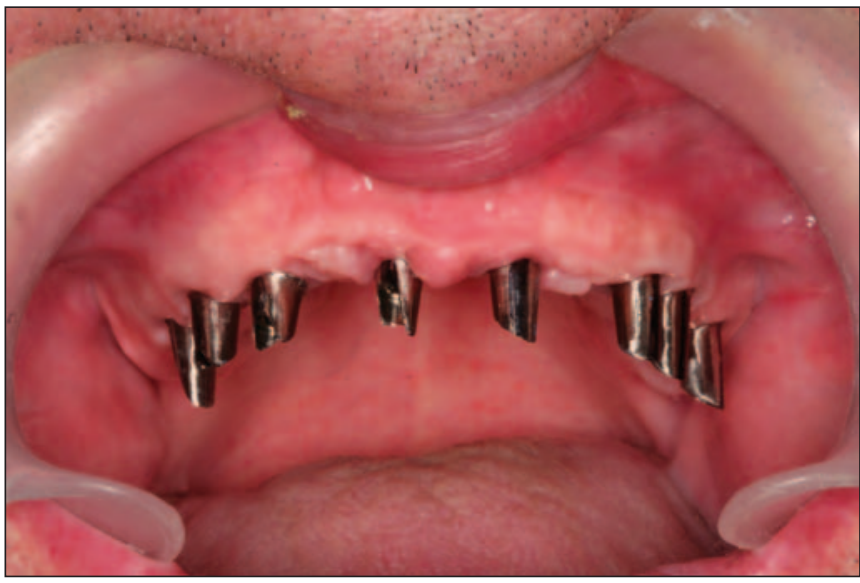

Fig. 7. Pilares mecanizados individualizados en el paciente geriátrico edéntulo total maxilar.

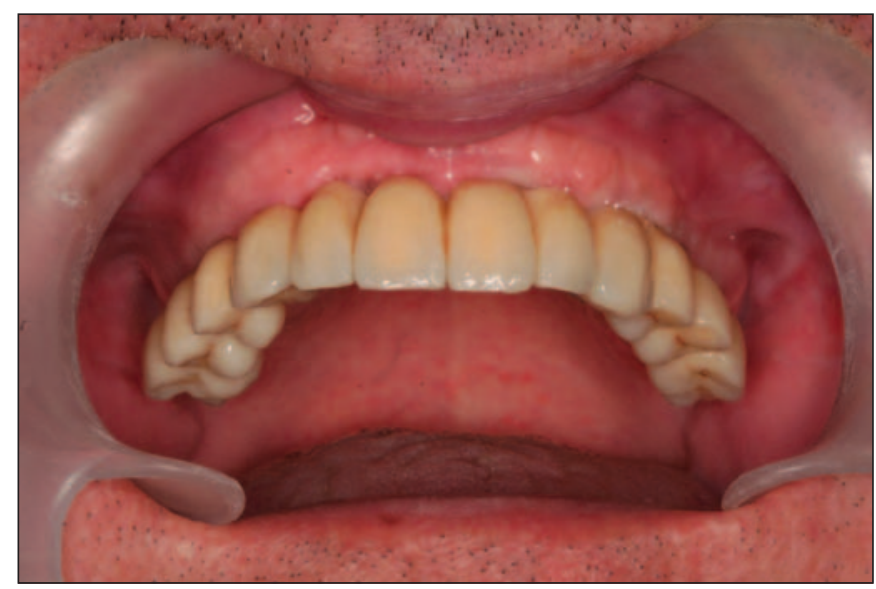

Fig. 8. Rehabilitación oral fija cementada en el paciente edéntulo maxilar 
es mayor conforme aumenta la longitud del mismo $(34,35)$. Sin embargo, se han realizado estudios de la inserción de implantes cortos en casos de reabsorción severa maxilar y/o mandibular que ofrecen la ventaja de una técnica quirúrgica relativamente sencilla y una escasa morbilidad. No obstante, la inserción de implantes cortos en casos de reabsorción ósea extrema ofrece la desventaja fundamental de que se compromete negativamente la ratio entre la longitud del implante y la distancia al plano oclusal provocando una biomecánica desfavorable (36).

El diámetro de los implantes constituye otro factor importante en el tratamiento implantológico porque un mayor diámetro puede incrementar la superficie de contacto entre el hueso y el implante. Es decir, que para la misma longitud los implantes con un diámetro más ancho presentan una mayor área de contacto óseo que los implantes de diámetro reducido $(34,35)$. La influencia de la longitud y del diámetro sobre el éxito de los implantes es confirmado en un estudio a 3 años con diferentes longitudes (7-16 $\mathrm{mm}$ ) y diámetros de implantes (3-4,9 mm), donde se destaca que el éxito fue del $90,7 \%$ para los implantes de 3-3,9 mm de diámetro y del $94,6 \%$ para los de 4-4,9 mm. La tasa de éxito fue del 66,7\% para los implantes de $7 \mathrm{~mm}$ de longitud y de $96,4 \%$ para los de $16 \mathrm{~mm}$ (37).

\section{PRÓTESIS SOBRE IMPLANTES}

La elección del tipo de rehabilitación del paciente edéntulo total va a estar relacionada con sus expectativas reales y con un grupo de factores orales, como son la cantidad y calidad del hueso, la relación intermaxilar, la estética facial y los factores oclusales. Desde un punto de vista extraoral, los antecedentes médicos, la capacidad funcional y el coste económico son también importantes. La posibilidad de sustituir dientes perdidos por estructuras protésicas sobre implantes ha representado una evolución muy positiva en el tratamiento de los pacientes totalmente desdentados $(1,3,4)$.

En este sentido, la incorporación de las sobredentaduras con implantes ha revolucionado la rehabilitación de los pacientes totalmente edéntulos. Las sobredentaduras implantosoportadas permiten la posibilidad de una unión directa de la prótesis a los implantes, y la transmisión de las fuerzas de los dientes artificiales al maxilar y/o la mandíbula asegurando no solamente la estabilidad de la prótesis, sino también disminuyendo los traumatismos sobre los tejidos blandos orales, y favoreciendo la sensación entre muchos pacientes de edad avanzada de que sus dientes artificiales han llegado a formar parte integral de ellos mismos, lo que ha aumentado, extraordinariamente, su calidad de vida oral (9). Entre los diversos tipos de prótesis sobre implantes en los pacientes edéntulos, las sobredentaduras ha representado un rápido incremento con una buena evidencia de resultados favorables. Esta realidad responde a cambios socioculturales y económicos de los adultos mayores, a la evolución de la odontología, a una mejor técnica prostodóncica, y a una mayor demanda estética y funcional por parte de estos pacientes ante el edentulismo total. El objetivo del tratamiento con sobredentaduras con implantes del paciente anciano edéntulo es mantener una rehabilitación oral favorable durante toda la vida sin una excesiva intervención por parte del profesional, salvo las medidas preventivas (4).

En pacientes mayores con un largo periodo de edentulismo total, tiene lugar una atrofia avanzada de los maxilares, por lo que una sobredentadura con la base acrílica puede proporcionar una buena opción terapéutica para corregir una gran discrepancia de las arcadas dentales, que soporte los tejidos vestibulares con un buen control salival y fonético, y favorezca un fácil acceso de higiene oral por el paciente adulto de edad avanzada $(4,5)$.

Un estudio español muestra la evaluación de los pacientes edéntulos totales geriátricos tratados con sobredentaduras mandibulares con implantes dentales mediante diferentes tipos de carga funcional (38). Fueron tratados 33 pacientes edéntulos totales con 127 implantes en la mandíbula para su rehabilitación prostodóncica con sobredentaduras. Todos los implantes fueron insertados en 1 cirugía y fueron cargados de forma convencional, precoz o inmediata. Después de un seguimiento clínico medio superior a 5 años, los resultados indican un éxito de los implantes del 96,9\% porque 4 implantes fracasaron. No hubo diferencias entre los diferentes protocolos de carga. El $100 \%$ de los pacientes fueron tratados 
con una sobredentadura implantosoportada, el $60,9 \%$ con retenedores de bolas y el 31,9\% mediante barras. El estudio indicaba que la rehabilitación oral de los pacientes edéntulos geriátricos con sobredentaduras mandibulares implantosoportadas mediante su carga convencional, precoz o inmediata representaba representar una terapéutica odontológica con éxito (38).

Los pacientes mayores edéntulos parciales y totales pueden ser tratados con prótesis fija con un éxito elevado. Es evidente que la prostodoncia fija es más estable, y proporciona un mejor bienestar frente a la sensación de pérdida física dental. Ahora bien, las prótesis fijas necesitan un mayor número de implantes, un reborde alveolar suficiente, un espacio interoclusal favorable y un mantenimiento más difícil que las sobredentaduras (Figuras 5-8) $(39,40)$. La valoración prostodóncica del paciente geriátrico edéntulo total que va a ser tratado mediante una rehabilitación fija sobre implantes es importante sobre todo cuando existe un edentulismo total en ambas arcadas superior e inferior. En estos casos, con un grado de reabsorción ósea importante que provoca un gran espacio interoclusal con una discrepancia notable entre ambos maxilares, puede obligar a elegir más conveniente una restauración híbrida con resina que una prótesis fija con porcelana, e incluso en casos muy desfavorables una sobredentadura (41).

El tratamiento con implantes mediante una rehabilitación fija maxilar o mandibular puede presentar resultados muy favorables tanto en pacientes edéntulos totales mayores como en pacientes adultos más jóvenes (39). En este sentido, un estudio sueco compara los resultados clínicos de 133 pacientes mayores de 80 años con 761 implantes insertados para 44 prótesis fijas maxilares y 95 prótesis fijas mandibulares con 115 adultos más jóvenes (41-79 años) con 670 implantes para 51 prótesis fijas maxilares y 67 prótesis fijas mandibulares (39). Los pacientes fueron seguidos durante un periodo de 5 años después de la carga funcional con una prótesis fija híbrida atornillada. El éxito acumulativo de los implantes fue similar en ambos grupos tanto en el maxilar (93\% y $92,6 \%$ ) como en la mandíbula $(99,5 \%$ y $99,7 \%)$. La complicación más frecuente entre los pacientes mayores de 80 años fue la mucositis, mientras que en los pacientes más jóvenes fue la fractura de la resina (39).

\section{IMPLANTOLOGÍA Y CALIDAD DE VIDA EN EL PACIENTE GERIÁTRICO}

El tratamiento con implantes dentales puede mejorar significativamente la calidad de vida de los pacientes mayores. La introducción de los implantes dentales en la rehabilitación de los pacientes mayores tratados con una prótesis completa convencional removible ha revolucionado su calidad de vida mejorando sus expectativas funcionales, estéticas y psicológicas (42-44).

En este sentido, cuando se compara la eficacia de una prótesis completa con una sobredentadura retenida con implantes en pacientes mayores, como demuestra un estudio turco, se demuestra desde un punto de vista funcional un mayor retención y fuerza de masticación en las prótesis mandibulares con implantes que en las prótesis completa removible, así como una mejora en la estética, el habla, y en el confort general (42).

Los efectos favorables sobre la masticación y la deglución son confirmados por un estudio brasileño donde se valoran a pacientes mayores de 60 años tratados con una prótesis fija sobre implantes (43). A lo largo de un periodo de 18 meses, los pacientes fueron evaluados a los 3, 6 y 18 meses después de la cirugía de implantes, demostrándose una importante reducción en las molestias dolorosas al masticar y deglutir, así como un descenso en la necesidad de ingestión de líquidos para mejorar la función oral (43).

La sustitución de la prótesis convencional por una prótesis sobre implantes puede ayudar a los pacientes geriátricos a modificar su dieta y mejorar su nutrición. En este sentido, un estudio demuestra que a los 6 meses de realizar un tratamiento con una sobredentadura mandibular, las medidas del estado nutricional en los pacientes mayores demuestran un incremento en parámetros antropométricos como son los pliegues cutáneos más densos en el bíceps, escápula y abdomen. Además, se muestra incrementos significativos sanguíneos de la albúmina, hemoglobina y vitamina B12 (44).

Las prótesis implantosoportadas pueden mejorar otras actividades de la vida diaria de los adultos mayores que influyen en su calidad de vida. Un estudio cana- 
diense revela que Las sobredentaduras mandibulares con implantes dentales mejoran, a los 2 meses, en relación a las prótesis completas convencionales, las actividades sociales y sexuales de los pacientes (44). Los resultados indicaron que este tipo de prótesis con implantes produce un impacto positivo en la comunicación social, la realización de actividades deportivas, relaciones afectivas y sexuales, así como en la prevención de la pérdida de las prótesis removibles durante la realización de estas actividades (45).

La estética es otro aspecto esencial que se beneficia de la rehabilitación oral con implantes en los adultos mayores. Los cambios estéticos pueden ser intraorales, como una mejor estética dental; y extraorales, como una mejor estética facial. La estética dental proporcionada por las prótesis sobre implantes constituye un aspecto esencial para incrementar el bienestar psicológico y social, que ha estado deteriorado durante muchos años en los pacientes edéntulos, insatisfechos con prótesis completas convencionales (46).

\section{CONCLUSIONES}

Actualmente, el tratamiento con implantes dentales constituye una alternativa odontológica muy importante en la rehabilitación prostodóncica de los pacientes mayores. El dentista necesita valorar las expectativas del paciente de acuerdo a su estado sistémico y oral, para realizar un diagnóstico y un plan de tratamiento individualizado. Las técnicas quirúrgica y prostodóncica debe ser establecidas de forma adecuada con las necesidades objetivas y subjetivas del paciente para conseguir unos resultados clínicos favorables tanto en el aspecto funcional como estético que mejoren significativamente su calidad de vida.

\section{BIBLIOGRAFÍA}

1. Velasco E, Velasco C, Monsalve L, Bullón P. Los implantes dentales no sumergidos en el paciente anciano. Rev Esp Geriatr Gerontol 2001;36:51-6.

2. Zarb G, Lekholm U, Albrektsson T, Tenenbaum H. Aging, osteoporosis and dental implants. Chicago: Quintessence, 2002.
3. Velasco E, Linares D, Velasco C, Monsalve L, Medel R. Las sobredentaduras con implantes oseointegrados en el paciente anciano. Av Perio Impl Oral 2003;15: 25-33.

4. Feine JS, Carlsson GE. Implant overdentures. The standard of care for edentulous patients. Chicago: Quintessence, 2003.

5. Stanford CM. Dental implants. A role in geriatric dentistry for the general practice? JADA 2007;138 (Suppl. 9):34S-40S.

6. Velasco E, España A, Ortiz I, Jiménez A. El tratamiento con implantes oseointegrados en gerodontología. En: Gerodontología. Sociedad Española de Gerodontología (ed.). Santiago de Compostela. 2012;243-53.

7. Lee HJ, Kim YK, Park JY, Kim SG, Kim MJ, Yun PY. Short-term clinical retrospective study of implants in geriatric patients older than 70 years. Oral Surg Oral Med Oral Pathol Oral Radiol Endod 2010;110:442-6.

8. Bryant SR, Zarb GA. Osseointegration of oral implants in older and younger adults. Int J Oral Maxillofac Implants 1998;13:492-9.

9. Vercruyssen M, Marcelis K, Coucke W, Naerte I, Quirynen $M$. Long-term, retrospective evaluation (implant and patient-centre outcome) of the twoimplants-supported overdenture in the mandible. Part 1: survival rate. Clin Oral Impl Res 2010;21:357-65.

10. Attard NJ, Zarb GA. Long-term treatment outcomes in edentulous patients with implant overdentures: the Toronto study. Int J Prosthodont 2004; 17:425-33.

11. Chrcarnovic BR, Albrektsson T, Wennerberg A. Diabetes and oral implant failure: asystematic review. J Dent Res 2014;93:859-67.

12. Holahan CM, Koka S, Kennel KA, Weaver AL, Assad DA, Regennitter FJ, Kademani D. Effect of osteoporotic status on the survival of titanium dental implants. Int J Oral Maxillofac Implants 2008;23:905-10. 
13. Khan AA, Morrison A, Hanley DA, Felsenberg D, McCauley LK, O’Ryan F, Reid IR et al. Diagnosis and management of osteonecrosis of the jaw: a systematic review and international consensus. J Bone Miner Res 2015;30:3-23.

14. Ihde S, Kopp S, Gundlach K, Konstantinovic VS. Effects of radiation therapy on craneofacial and dental implants: a review of the literature. Oral Surg Oral Med Oral Pathol Oral Radiol Endod 2009;107:55-65.

15. Anderson L, Meraw S, Al-Hezaimi K, Wang HL. The influence of radiation therapy on dental implantology. Impl Dent 2012;22:31-8.

16. Packer M, Nikitin V, Coward T, Davis DM, Fiske J. The potential benefits of dental implants on the oral health quality of life of people with Parkinson's disease. Gerodontology 2009;26:11-8.

17. Faggion Jr CM. Critical appraisal of evidence supporting the placement of dental implants in patients with neurodegenerative diseases. Gerodontology. En prensa.

18. Strietzel FP, Reichart PA, Kale A, Kulkarni M, Wegner B, Küchler I. Smoking interferes with the prognosis of dental implant treatment: a systematic review and metaanalisis. J Clin Periodontol 2007; 34:523-44.

19. Alman AC, Johnson LR, Calverley DC, Grunwald GK, Lezotte DC, Harwood JEF, Hokanson JE. Loss of alveolar bone due to periodontal disease exhibits a threshold on the association with coronary heart disease. J Periodontol 2011;82: 1304-13.

20. Marrone A, Lasserre J, Bercy P, Brecx MC. Prevalence and risk factors for peri-implant disease in Belgian adults. Clin Oral Impl Res 2013; 24:934-40.

21. Isaksson R, Becktor J, Brown A, Laurizohn C, Isaksson $\mathrm{S}$. Oral health and oral implant status in edentulous patients with implant-supported dental prostheses who are receiving long-term nursing care. Gerodontology 2009;26:245-9.
22. Visser A, de Baat C, Hoeksema AR, Vissink A. Oral implants in dependent elderly persons: blessing or burden? Gerodontology 2011;28:7680.

23. Kimura T, Wada M, Suganami T, Miwa S, Hagiwara Y, Maeda Y. Dental implant status of patients receiving long-term nursing care in Japan. Clin Impl Dent Relat Res 2015;17(Suppl. 1): e163-7.

24. Morita I, Nagasaki H, Kato K, Murakami T, Tsuboi S, Hayashizaki J, et al. Relationship between survival rates and numbers of teeth in an elderly Japanese population. Gerodontology 2006;29:214-8.

25. Kowar J, Stenport V, Jemt T. Mortality patterns in partially edentulous and edentulous elderly patients treated with dental implants. In J Prosthodont 2014;27:250-6.

26. Friberg B, Jemt T. Rehabilitation of edentulous mandibles by means of osseointegrated implants: a 5-year follow-up study on one or two-stage surgery, number of implants, implant surfaces, and age at surgery. Clin Impl Dent Relat Res. En prensa.

27. Pérez O, Velasco E, González L, García A, Rodríguez $\mathrm{O}$. Técnicas quirúrgicas complejas en el tratamiento con implantes oseointegrados del maxilar superior. Av Perio Impl Oral 2006;18:10-9.

28. Leiser Y, Rachmiel A. Bilateral sinus lift augmentation in elderly patients with severely atrophic maxilla. Oral Health Dent Manag 2014;13:11615.

29. Nkenke E, Neukam FW. Autogenous bone harvesting and grafting in advances jaw resorption: morbidity, resorption and implant survival. Eur J Oral Impl 2014;7(Suppl. 2): S20317.

30. Velasco E, Segura JJ, Linares D, Medel R, Poyato M. La carga inmediata de los implantes transicionales en sobredentaduras mandibulares en adultos mayores. Av Perio Impl Oral 2004;16:10713. 
31. Velasco E. Implantología oral guiada asistida por ordenador. Fundamentos científicos y práctica clínica. Madrid: Ripano, 2013.

32. Mericske-Stern R, Worni A. Optimal number of oral implants for fixed reconstructions: a review of the literature. Eur J Oral Implantol 2014;7(Suppl. 2):S133-S53.

33. Brunski JB. Biomechanical aspects of the optimal number of implants to carry a cross-arch full restoration. Eur J Oral Implantol 2014;7(Suppl. 2): S111-S31.

34. Renouard F, Nisand D. Impact of implant length and diameter on survival rates. Clin Oral Impl Res 2006;17 (Suppl. 2):35-51.

35. Lee JH, Frias V, Lee KW, Wright RF. Effect of implant size and shape on implant success rates: a literature review. J Prosthet Dent 2005;94:377-81.

36. Annibali S, Cristalli MP, Dell'Aquila D, Bignozzi I, La Monaca G, Pilloni A. Short dental implants: A systematic review. J Dent Res 2012;91:25-32.

37. Winkler S, Morris HF, Ochi S. Implant survival to 36 months as related to length and diameter. Ann Periodontol 2000;5:22-31.

38. Linares D, Medel R, Segura JJ, Poyato M, Velasco E. La eficacia clínica de la carga convencional, precoz e inmediata en sobredentaduras mandibulares con implantes dentales. Un estudio comparativo a 5 años. Av Perio Impl Oral 2010; 22:11-8.

39. Engfors I, Ortorp A, Jemt T. Fixed implantsupported prostheses in elderly patients: a 5-year retrospective study of 133 edentulous patients older than 79 years. Clin Impl Dent Relat Res 2004; 6:190-8.

40. Kowar J, Eriksson A, Jemt T. Fixed implantsupported prostheses in elderly patients: a 5-year retrospective comparison between partially and completely edentulous patients aged 80 years or older at implant surgery. Clin Impl Dent Relat Res 2013;15:37-46.
41. Velasco E, García A, Pato J, Cruz JM, Lorrio JM. El tratamiento con implantes en pacientes edéntulos totales mandibulares mediante rehabilitación fija. Av Perio Impl Oral 2007;19:151-9.

42. Geckilli O, Bilhan H, Mumcu E, Dayan C, Yabul A, Tuncer N. Comparison of patient satisfaction, quality of life, and bite force between elderly edentulous patients wearing mandibular two implant-supported overdentures and conventional complete dentures after 4 years. Spec Care Dentist 2012;32:136-41.

43. Berretin-Felix G, Machado WM, Genaro KF, Filho HN. Effects of mandibular fixed implantsupported prostheses on masticatory and swallowing functions in completely edentulous elderly individuals. Int J Oral Maxillofac Implants 2009;24:110-7.

44. Morais JA, Heydecke G, Pawliuk J, Lund JP, Feine JS. The effects of mandibular two-implant overdentures on nutrition in elderly edentulous individuals. J Dent Res 2003;82:53-8.

45. Heydecke G, Thomason JM, Lund JP, Feine JS. The impact of conventional and implant supported prostheses on social and sexual activities in edentulous adults. Results from a randomized trial 2 months after treatment. J Dent 2005;33:649-57.

46. Turkylmaz I, Company AM, McGlumphy EA. Should edentulous patients be constrained to removable complete dentures? The use of dental implants to improve the quality of life for edentulous patients. Gerodontology 2010;27:310 .

\section{CORRESPONDENCIA}

Prof. Eugenio Velasco Ortega

Facultad de Odontología

Avicena, s/n

41009 Sevilla

Correo electrónico: evelasco@us.es 